\title{
Impact of the sleep apnea management group clinic on positive airway pressure adherence
}

\author{
Katie Tran ${ }^{1} \cdot$ Lu Wang $^{2} \cdot$ Reena Mehra ${ }^{3} \cdot$ Robon Vanek $^{3} \cdot$ Shivani Kaw $^{3} \cdot$ Tina Campean $^{3} \cdot$ Nancy Foldvary-Schaefer $^{3}$. \\ Douglas E. Moul ${ }^{3} \cdot$ Harneet Walia ${ }^{3}$
}

Received: 2 November 2020 / Revised: 26 February 2021 / Accepted: 15 March 2021 / Published online: 29 April 2021

(C) The Author(s), under exclusive licence to Springer Nature Switzerland AG 2021

\begin{abstract}
Background Positive airway pressure (PAP) adherence is critical for managing obstructive sleep apnea (OSA). We postulate that group-based Sleep Apnea Management (SAM) clinic, which harnesses the benefits of providing mutual support as well as facilitates access to system-based resources and education, will confer improvements in PAP adherence.

Methods Data from SAM clinic attendees from January 2017 to June 2018 were retrospectively analyzed. Adherence data at SAM baseline visit and 1-3 months follow-up were collected. Average PAP usage from all-days and days used were analyzed along with demographics, co-morbidities, and Epworth Sleepiness Scale. Adherence was defined as $\geq 4$ hours a night for $\geq 70 \%$ of nights over a 30-day period. Key structural elements of the SAM group clinic model were co-presence of the OSA care team members and peer group support. Key efficiency elements were group education and the prompt-to-patient multidisciplinary troubleshooting adherence barriers.

Results Of 110 SAM clinic attendees, average age was $60.9 \pm 12.7$ years, $53 \%$ were men, and $46 \%$ Caucasian. At baseline, the mean for average-all-days usage was 4.2 hours, mean average-days-used usage was 5.2 hours, and mean percentage-of-days usage $\geq 4$ hours was $57 \%$. At follow-up, the mean average-all-days usage increased 1.2 hours $(p<0.001)$, mean average-days-used usage increased 0.8 hours $(p<0.001)$, and the mean percentage-of-days with usage $\geq 4$ hours increased $16 \%(p<0.001)$. At baseline, $46 \%$ of patients met criteria for adherence, which increased to $66 \%$ at follow-up.

Conclusion In this study, after the SAM clinic, all PAP adherence parameters improved significantly. This observational study serves as a proof of concept study for future trials pertaining to group clinic in managing PAP adherence in OSA.
\end{abstract}

Keywords Positive airway pressure $\cdot$ Obstructive sleep apnea $\cdot$ Group clinic $\cdot$ Adherence

\section{Introduction}

Obstructive sleep apnea (OSA) is a common sleep-related breathing disorder that affects about $10-15 \%$ of the general adult population [1]. It has been associated with many negative health outcomes including cardiovascular disease, stroke, cognitive decline, and an increase in mortality [2-4]. Hence,

Harneet Walia

Harneet_walia@yahoo.com

1 Center for Sleep and Breathing Disorders, UT Southwestern University, Dallas, TX, USA

2 Department of Quantitative Health Sciences, Cleveland Clinic, Cleveland, OH, USA

3 Sleep Disorders Center, Neurological Institute, Cleveland Clinic, Cleveland, OH, USA
OSA treatments are of substantial relevance for secondary prevention interventions in population health. The mainstay treatment for OSA is with positive airway pressure (PAP) therapy in moderate to severe cases. Although it is highly effective, its full potential to mitigate the negative consequences of untreated OSA in populations is often limited by impaired treatment adherence. There have been many studies examining the factors that affect PAP adherence as well as interventions to improve it, but it remains a pertinent issue in OSA treatment [5-12]. Adherence is a common barrier, not only in OSA treatment, but also in the treatment of other medical conditions as well. Group-based clinics have demonstrated effective results in educating and improving adherence to therapies across various medical conditions including obesity, diabetes, and heart failure [13-16]. These clinics can provide patients with a degree of support, education, and motivation that is not available in a typical individualized clinical 
visit. However, the effectiveness of group clinics on PAP adherence remains to be characterized.

At the Cleveland Clinic Sleep Disorders Center, the Sleep Apnea Management (SAM) clinic is a group-based clinic to provide patients with OSA greater accessibility to treatment. Patients are referred to SAM clinics both by sleep and nonsleep providers. Reasons for referrals were mainly focused on PAP adherence, but also included ongoing management of OSA. The multidisciplinary visit team focuses on the direct troubleshooting of technical issues and addresses any additional barriers that may be limiting treatment adherence. With adherence being a major limitation to the treatment of OSA, we have synergized group-based dynamics to improve PAP adherence, but have not yet formally studied outcome metrics from this SAM clinic process.

The objective of this study was to assess the impact of the SAM clinic on PAP adherence. We postulate that there would be a statistically significant improvement in overall PAP usage from baseline to follow-up after attending the SAM clinic.

\section{Methods}

\section{Study population}

Cleveland Clinic electronic medical record data were used to identify and select the patient population. Inclusion criteria included patients over the age of 18 years who attended the SAM clinic from January 2017 to June 2018 and who also had a follow-up appointment in the SAM clinic 1-3 months after their baseline visit. Exclusion criteria included those who were less than 18 years old and those who did not attend a followup SAM appointment. The pre-treatment/post-treatment comparison was the focus of this study.

\section{Measurements and definitions}

Demographics included age, sex, race, and body mass index (BMI). Median household income was determined by zip code. Comorbid conditions such as hypertension, congestive heart failure, coronary artery disease, atrial fibrillation, chronic obstructive pulmonary disease, asthma, gastroesophageal reflux disease, seizures, stroke, depression, anxiety, diabetes mellitus, and thyroid disorders were obtained through the medical record. The Epworth Sleepiness Scale (ESS) was used as a self-report measure for assessing sleepiness [17]. The ESS asks respondents about their relative tendency to doze in 8 standard daily life situations, with a total score ranging from 0 to 24 .

Patients' most recent diagnostic sleep study data from the time of baseline SAM clinic visit included date of the study, total sleep time, sleep efficiency, apnea-hypopnea index (AHI), supine index, rapid eye movement (REM) index, oxygen saturation $(\mathrm{SpO} 2)$ nadir, and time spent with $\mathrm{SpO} 2$ $<90 \%$. The diagnostic sleep study could be from an in-lab polysomnogram or a home sleep apnea test.

The date of PAP set-up by the patient's durable medical equipment (DME) company was also collected. PAP usage data was obtained from modem downloads. Usage over a 30day period was divided into average (all-days) usage, average (days-used) usage, and percentage-of-days usage $\geq 4$ hours. Baseline data was obtained at the first SAM clinic visit time point and again at the 1-3 months follow-up SAM clinic visit time point. Adherence was defined as $\geq 4$ hours a night for $\geq 70 \%$ of nights over a 30 -day period, per Centers for Medicare and Medicaid Services (CMS) criteria.

\section{Sleep apnea management clinic visit process}

This group-based clinic visit included a sleep medicine provider (sleep medicine physician or nurse practitioner), and usually one or two representatives from a DME company or a nurse proxy who is knowledgeable with PAP intricacies. About 6-10 patients were scheduled at a time. Patients were instructed to arrive 15 minutes early to check-in and fill out a questionnaire about their current machine and any issues they would like to discuss regarding therapy including mask leak, skin irritation, improper mask fit, claustrophobia, nasal congestion, nose or mouth dryness, aerophagia, pressure intolerance, or any other concerns they may have regarding therapy. They were also instructed to bring in their PAP machine and supplies to the visit. The sleep medicine provider led the group, with all group members able to participate (e.g., a DME representative might be asked a technical question about PAP machines). Each session began with a brief educational discussion about OSA and PAP therapy. After that discussion, the provider then interacted with each patient personally to assess any individual issues interfering with PAP adherence. DME representatives were asked to intervene and help troubleshoot for individual patients during that visit if relevant, such as changing pressure settings and adjusting mask or headgear straps. Patients were encouraged to ask questions, share their experiences, and offer support as they felt comfortable. The provider formulated an individualized follow-up plan for each patient which may include another SAM visit or a referral back to a sleep medicine specialist for more complicated issues. The SAM clinic is set up as a weekly recurring visit. Follow-up visits are based on individual need so each group varies from visit to visit. In this analysis, the whole treatment session is considered to be the therapeutic exposure under investigation.

\section{Statistical analysis}

Continuous variables are presented as mean \pm standard deviations or medians and quartiles. Categorical variables are 
presented as frequency and percentages. Comparisons between groups were performed with two-sample t-test or Kruskal-Wallis test for continuous variables based on distribution and Pearson's chi-square test or Fisher's exact test for categorical variables. A linear mixed-effects (LME) model was used to account for correlation of repeated PAP adherence measures on the same patient assuming compound symmetry correlation structure. Two time points were treated as a categorical fixed factor to assess the change of adherence measures from baseline to follow-up. The interaction of time and baseline adherence was tested to evaluate the difference in the change between adherent and non-adherent groups. Covariates adjusted in models included age, gender, race, median household income, and baseline AHI. All data were imported into SAS 9.4 (The SAS Institute Cary, NC) and were based on a 0.05 level of significance.

\section{Results}

Of the 128 patients in the sample population, 18 were excluded for insufficient data or were lost to follow-up. The analytic sample comprised of 110 patients. A slight majority were men $(53 \%)$. The average age was $60.9 \pm 12.7$ years. Caucasian and African American patients were about equally represented. Average BMI was $34.3 \pm 7.2$. The median ESS score was 10 $[5,14]$. The median AHI was $27.3[15.5,44.9]$. A majority had a cardiovascular comorbid condition, with hypertension being the most common (50\%). The average time from first CPAP usage to the first SAM clinic was about 1.7 years. Overall, there was no difference between the two groups at baseline (Table 1).

At baseline, the estimated mean for average (all-days) usage was 4.23 hours; while that for average (days-used) usage was 5.20 hours, and the percentage-of-days usage $\geq 4$ hours was $56.7 \%$. At $1-3$ months follow-up, the mean in average (all-days) usage was 5.41 hours, increased by 1.18 hours $(p<0.001)$, while for the mean of average (days-used) usage was 6.04 hours, increased by 0.83 hours $(p<0.001)$, and the mean percentage-of-days with usage $\geq 4$ hours was $73.04 \%$, increased $16.34 \%(p<0.001)$ (Table 2). At baseline, $46 \%$ met the criteria of adherence, while this number improved to $66 \%$ at follow-up.

Those who did not meet adherence criteria at baseline had an improvement in average (all-days) usage by 2.2 hours and an improvement in average (days-used) usage by 1.6 hours at follow-up, compared with a stable usage in patients met adherence criteria at baseline. Though age, gender, race, median household income, and baseline AHI were adjusted in the LME model, no factor was significantly associated with average PAP usage, either for all-days or days-used. Those who had met adherence criteria at baseline had less increase in overall usage (Table 2). Among the 59 patients who were non-adherent at baseline, $47.5 \%$ achieved adherence at follow-up. Of those who were adherent at baseline $(\mathrm{N}=51)$, $88.2 \%$ retained adherence.

\section{Discussion}

In this retrospective observational study, we assessed PAP adherence after utilization of a group clinic designed to educate, motivate, and troubleshoot issues on PAP adherence. Patients experienced significant improvement in overall PAP usage from baseline after attending the group-based SAM clinic. At follow-up, $66 \%$ of patients met adherence criteria and there was greater improvement seen in those who were initially non-adherent. Although the PAP adherence improves in the overall group of patients, when studying the groups separately, it is the non-adherent group where adherence improved more significantly.

Although there is less time allotted per patient by the sleep medicine provider compared to a usual visit, the SAM clinic visits synergistically provided an accessible, effective, and efficient means to improving PAP adherence. Providers were able to educate and answer questions, but the patients could also get support and encouragement from their peers. From other literature, obtaining peer support may have unique treatment effectiveness [18]. One study about sleep apnea treatment comparing a one-time group educational session versus individual sessions not only found that group education improved adherence, but also increased acceptance of therapy with decreased time to treatment [19]. However, this was a one-time session and some patients may need ongoing education and treatment. Another study showed improvement in PAP usage by providing a 2-hour group CPAP clinic scheduled every 6 months over a 2-year period [20].

Compared to other group approaches, the specific design of the SAM clinic is unique in that it is an ongoing weekly or biweekly billable clinical visit that maximizes accessibility for prompt problem solving. With the multidisciplinary approach, certain issues that may be difficult to handle in an individual clinic may be conveniently addressed in the SAM clinic with the help of the DME representatives (e.g., special information, mask fitting, and setting changes). The DME representatives can use the SAM clinic as their own visit too, in coordination with the providers more immediate directives. This arrangement is better than having an order placed to the DME company and then waiting to hear from them to make necessary changes. The clinic was not limited to only those who were non-adherent, but was also available to those in need of annual follow-up or for adherence check for insurance purposes. The varied case mix in the SAM clinic tends to normalize PAP adherence for patients having trouble with adherence. Because it is run as a regular office visit, the follow-ups are scheduled based on the clinician's judgment. Some patients 
Table 1 Comparison of baseline characteristics by baseline percent day $\geq 4 \mathrm{hr}$ use $<70 \%$ vs. $\geq 70 \%$

\begin{tabular}{|c|c|c|c|c|}
\hline Factors & Total $(\mathrm{N}=110)$ & $<70 \%(\mathrm{~N}=59)$ & $\geq 70 \%(\mathrm{~N}=51)$ & $\begin{array}{l}p- \\
\text { value }\end{array}$ \\
\hline Age & $60.9 \pm 12.7$ & $60.0 \pm 14.1$ & $62.0 \pm 11.0$ & $0.42^{\mathrm{a}}$ \\
\hline Gender (men), \% & 52.7 & 49.2 & 56.9 & $0.42^{\mathrm{c}}$ \\
\hline BMI, $\mathrm{kg} / \mathrm{m}^{2}$ & $34.3 \pm 7.2$ & $34.8 \pm 5.7$ & $33.7 \pm 8.6$ & $0.41^{\mathrm{a}}$ \\
\hline Ethnicity (Hispanic or Latino), \% & 4.5 & 3.4 & 5.9 & $0.66^{\mathrm{d}}$ \\
\hline Race, $\%$ & & & & $0.090^{\mathrm{c}}$ \\
\hline White & 46.4 & 37.3 & 56.9 & \\
\hline Black & 46.4 & 55.9 & 35.3 & \\
\hline Other & 7.3 & 6.8 & 7.8 & \\
\hline Epworth Sleepiness Scale Total & $10.0[5.0,14.0]$ & $10.0[5.0,14.0]$ & $9.0[5.0,13.0]$ & $0.50^{\mathrm{b}}$ \\
\hline Median household income $2016(\$ 1000)$ & $52.2[32.1,18.8]$ & $52.7[32.1,66.6]$ & $51.6[32.1,72.2]$ & $0.58^{\mathrm{b}}$ \\
\hline Years from the first use of CPAP & $1.7[0.19,4.7]$ & $1.8[0.26,5.1]$ & $1.2[0.19,4.2]$ & $0.48^{\mathrm{b}}$ \\
\hline \multicolumn{5}{|l|}{ Polysomnogram } \\
\hline Total sleep time (TST), hours & $5.5 \pm 1.4$ & $5.6 \pm 1.6$ & $5.5 \pm 1.2$ & $0.79^{\mathrm{a}}$ \\
\hline Sleep efficiency, $\%$ & $76.0[63.0,83.4]$ & $75.3[58.8,82.8]$ & $76.7[64.7,84.3]$ & $0.40^{\mathrm{b}}$ \\
\hline AHI, events/hour & $27.3[15.5,44.9]$ & $27.7[15.1,45.3]$ & $25.3[16.2,44.9]$ & $0.89^{\mathrm{b}}$ \\
\hline AHI in supine position, events/hour & $37.6[19.4,67.9]$ & $30.1[19.4,62.5]$ & $40.3[18.8,69.2]$ & $0.85^{\mathrm{b}}$ \\
\hline AHI in REM sleep, events/hour & $48.0[22.9,65.8]$ & $48.0[26.5,63.2]$ & $47.6[16.7,71.6]$ & $0.88^{\mathrm{b}}$ \\
\hline Minimum $\mathrm{SaO} 2, \%$ & $82.0[77.0,87.0]$ & $82.0[78.0,87.0]$ & $82.0[73.0,85.0]$ & $0.29^{\mathrm{b}}$ \\
\hline Mean $\mathrm{SaO} 2, \%$ & $93.0[92.0,94.0]$ & $93.0[92.0,94.0]$ & $93.0[91.0,95.0]$ & $0.66^{\mathrm{b}}$ \\
\hline Total time $\mathrm{SaO} 2<90 \%$, minutes & $7.1[1.1,28.4]$ & $6.1[0.50,28.8]$ & $9.8[2.1,28.4]$ & $0.33^{\mathrm{b}}$ \\
\hline Percentage of time $\mathrm{SaO} 2<90 \%$ & $3.9[0.60,12.8]$ & $3.7[0.20,15.0]$ & $4.5[0.80,12.8]$ & $0.51^{\mathrm{b}}$ \\
\hline \multicolumn{5}{|l|}{ Comorbid Conditions } \\
\hline Hypertension & $55(50.0)$ & $31(52.5)$ & $24(47.1)$ & $0.57^{\mathrm{c}}$ \\
\hline Congestive heart failure & $7(6.4)$ & $5(8.5)$ & $2(3.9)$ & $0.45^{\mathrm{d}}$ \\
\hline Coronary artery disease & $11(10.0)$ & $7(11.9)$ & $4(7.8)$ & $0.48^{\mathrm{c}}$ \\
\hline Atrial fibrillation & $5(4.5)$ & $2(3.4)$ & $3(5.9)$ & $0.66^{\mathrm{d}}$ \\
\hline Chronic obstructive pulmonary disease & $2(1.8)$ & $1(1.7)$ & $1(2.0)$ & $0.99^{\mathrm{d}}$ \\
\hline Asthma & $11(10.0)$ & $7(11.9)$ & $4(7.8)$ & $0.48^{\mathrm{c}}$ \\
\hline Gastroesophageal reflux disease & $22(20.0)$ & 11(18.6) & $11(21.6)$ & $0.70^{\mathrm{c}}$ \\
\hline Depression & $19(17.3)$ & $9(15.3)$ & $10(19.6)$ & $0.55^{\mathrm{c}}$ \\
\hline Anxiety & $9(8.2)$ & $5(8.5)$ & $4(7.8)$ & $0.99^{\mathrm{d}}$ \\
\hline Diabetes mellitus type 2 & $21(19.1)$ & $12(20.3)$ & $9(17.6)$ & $0.72^{\mathrm{c}}$ \\
\hline Hypothyroidism & $8(7.3)$ & $4(6.8)$ & $4(7.8)$ & $0.99^{\mathrm{d}}$ \\
\hline
\end{tabular}

Statistics presented as Mean $\pm \mathrm{SD}$, Median [P25, P75] or $N$ (column \%). $p$-values: a=two-sample $\mathrm{t}$ test, $\mathrm{b}=$ Kruskal-Wallis test, $\mathrm{c}=$ Pearson's chi-square test, $\mathrm{d}=$ Fisher's Exact test may need to be seen more often than others. This provided an individualistic approach by accommodating to the individual patient's needs.

Limitations to this study include the following: (1) this was a retrospective study without a control group which limits the conclusions drawn from our analysis; (2) our follow-up schedule was not standardized which may add variability to the outcome; (3) the type of patients seen in the SAM clinic was not limited to only those who struggled with adherence or those who were only recently diagnosed with OSA which may reduce the generalizability of our results; (4) while long-term adherence may be predicted by short-term adherence, we only measured PAP adherence 1-3 months after attending the SAM clinic and not necessarily after initial PAP set-up. The average time from initial PAP set-up to the first SAM clinic was 1.7 years which allows for greater variability in a patient's level of education on PAP usage. On the other hand, this study was more focused on effectiveness in a usual practice setting, because the follow-ups were scheduled, based on an individualized plan. It should be noted that our results should not be extrapolated to mild OSA cases. 
Table 2 Change in PAP Adherence $(\mathrm{N}=110)$

\begin{tabular}{|c|c|c|c|c|}
\hline & Baseline $[95 \% \mathrm{CI}]$ & $1-3$ months $[95 \% \mathrm{CI}]$ & Mean change $[95 \% \mathrm{CI}]^{*}$ & $p$ value of change \\
\hline \multicolumn{5}{|c|}{ Average usage (all days) - hours, minutes $\$$} \\
\hline Overall & 4hrs $14 \mathrm{~min}$ [3hr 44min, 4hrs $43 \mathrm{~min}]$ & $5 \mathrm{hrs} 25 \mathrm{~min}$ [4hrs $55 \mathrm{~min}, 5 \mathrm{hrs} 54 \mathrm{~min}$ ] & $1 \mathrm{hrs} 11 \mathrm{~min}$ [46min, $1 \mathrm{hr} 35 \mathrm{~min}]$ & $<0.001$ \\
\hline Non-adherent & $2 \mathrm{hrs} 8 \mathrm{~min}$ [1 $\mathrm{hr} 40 \mathrm{~min}, 2 \mathrm{hrs} 35 \mathrm{~min}]$ & 4hrs $19 \mathrm{~min}$ [3hrs $51 \mathrm{~min}, 4 \mathrm{hrs} 47 \mathrm{~min}]$ & $2 \mathrm{hrs} 11 \mathrm{~min}$ [1 $\mathrm{hr} 43 \mathrm{~min}, 2 \mathrm{hrs} 40 \mathrm{~min}]$ & $<0.001$ \\
\hline Adherent & $6 \mathrm{hrs} 41 \mathrm{~min}$ [6hrs $12 \mathrm{~min}, 7 \mathrm{hrs} 11 \mathrm{~min}]$ & $6 \mathrm{hrs} 42 \mathrm{~min}$ [6hrs $13 \mathrm{~min}, 7 \mathrm{hrs} 12 \mathrm{~min}]$ & $0.6 \mathrm{~min}[-30.6 \mathrm{~min}, 31.8 \mathrm{~min}]$ & 0.97 \\
\hline \multicolumn{5}{|c|}{ Average usage (days used) - hours, minutes $\$$} \\
\hline Overall & $5 \mathrm{hrs} 12 \mathrm{~min}$ [4hrs $46 \mathrm{~min}, 5 \mathrm{hrs} 38 \mathrm{~min}]$ & $6 \mathrm{hrs} 2 \mathrm{~min}$ [5hrs $36 \mathrm{~min}, 6 \mathrm{hrs} 28 \mathrm{~min}]$ & $50 \mathrm{~min}[26 \mathrm{~min}, 1 \mathrm{hr} 14 \mathrm{~min}]$ & $<0.001$ \\
\hline Non-adherent & $3 \mathrm{hrs} 43 \mathrm{~min}[3 \mathrm{hrs} 13 \mathrm{~min}, 4 \mathrm{hrs} 13 \mathrm{~min}]$ & $5 \mathrm{hrs} 16 \mathrm{~min}$ [4hrs $46 \mathrm{~min}, 5 \mathrm{hrs} 46 \mathrm{~min}]$ & $1 \mathrm{hr} 34 \mathrm{~min}[1 \mathrm{hr} 2 \mathrm{~min}, 2 \mathrm{hr} 5 \mathrm{~min}]$ & $<0.001$ \\
\hline Adherent & $6 \mathrm{hrs} 57 \mathrm{~min}$ [6hrs $25 \mathrm{~min}, 7 \mathrm{hrs} 29 \mathrm{~min}]$ & $6 \mathrm{hrs} 57 \mathrm{~min}$ [6hrs $25 \mathrm{~min}, 7 \mathrm{hrs} 29 \mathrm{~min}]$ & $0.0 \mathrm{~min}[-0.34 \mathrm{~min}, 33.6 \mathrm{~min}]$ & 0.99 \\
\hline \multicolumn{5}{|c|}{ Percentage of days with usage $\geq 4$ hours $(\%)$} \\
\hline Overall & $56.70[50.48,62.92]$ & $73.04[66.82,79.25]$ & $16.34[10.45,22.22]$ & $<0.001$ \\
\hline
\end{tabular}

*Least square means and $95 \%$ confidence intervals of the difference from baseline to 1-3 months follow-up

${ }^{\$}$ Mean change had statistical significant difference between adherent and non-adherent group on average usage all days and average usage days used $(p<0.001$ for both)

This was a proof of concept study that illustrated the positive impact and effectiveness the SAM clinic had on improving PAP adherence, especially in those who struggled with adherence at baseline. Because there was no control group, future prospective studies with a control group and long-term follow-up are needed, along with components-of-treatment analysis, to fully optimize the impact of this group treatment model. For this reason, the Cleveland Clinic Sleep Disorders Center has begun recruitment for a randomized controlled trial involving the SAM clinic model, set to conclude in 2021.

The SAM clinic model is one in the family of treatment models based in the social sciences, nursing, and public health literature. It operates logistically like a regular individual office clinic, but organizes components of care synergistically to provide an effective, efficient, and well-rounded approach to PAP adherence and OSA management.

This retrospective review was conducted months prior to the global pandemic of COVID-19, which has since changed some of the ways of healthcare including in-person clinics. We understand that group visits at this moment in time would not be wise nor responsible. Our model for the SAM clinic has since changed to virtual group visits with smaller group numbers overall; however, the principle practice remains the same and we suspect continued success in improving patients' overall adherence.

Abbreviations AHI, apnea-hypopnea index; BMI, body mass index; DME, durable medical equipment; ESS, Epworth Sleepiness Scale; OSA, obstructive sleep apnea; PAP, positive airway pressure; REM, rapid eye movement; SAM, Sleep Apnea Management

Authors' contribution All authors have seen and approved the manuscript.

\section{Declarations}

Conflict of interest 1. Katie Tran, M.D - no financial disclosures, no conflicts of interests

2. Lu Wang, M.S - no financial disclosures, no conflicts of interests

3. Reena Mehra, M.D, M.S - her institution has received positive airway pressure machines and equipment from Philips Respironics for use in NIH-funded research. She has received honorarium from the American Academy of Sleep Medicine for speaking

4. Robon Vanek, R.N, A.N.P—no financial disclosures, no conflicts of interests

5. Shivani Kaw - no financial disclosures, no conflicts of interests

6. Douglas E Moul, M.D., M.P.H.- no financial disclosures, no conflicts of interests

7. Tina Campean, R.N-no financial disclosures, no conflicts of interests

8. Nancy Foldvary-Schaefer, D.O, M.S - is a consultant for Jazz Pharmaceuticals

9. Harneet Walia, M.D — has received funding from Resmed for randomized controlled trial to assess the impact of sleep apnea management clinic in PAP adherence. She is the principal investigator of the studies with hypoglossal nerve stimulator.

Ethics approval and consent to participate All procedures performed in studies involving human participants were in accordance with the ethical standards of the institutional and/or national research committee and with the 1964 Helsinki declaration and its later amendments or comparable ethical standards. This was a retrospective study; informed consent was not obtained.

\section{References}

1. Punjabi NM (2008) The epidemiology of adult obstructive sleep apnea. Proc Am Thorac Soc 5(2):136-143. https://doi.org/10.1513/ pats.200709-155MG

2. Shamsuzzaman ASM, Gersh BJ, Somers VK (2003) Obstructive sleep apnea: implications for cardiac and vascular disease. JAMA. 290(14):1906-1914. https://doi.org/10.1001/jama.290.14.1906

3. Yaggi HK, Concato J, Kernan WN, Lichtman JH, Brass LM, Mohsenin V (2005) Obstructive sleep apnea as a risk factor for 
stroke and death. $N$ Engl J Med 353(19):2034-2041. https://doi. org/10.1056/NEJMoa043104

4. Bawden FC, Oliveira CA, Caramelli P (2011) Impact of obstructive sleep apnea on cognitive performance. Arq Neuropsiquiatr 69(4): 585-589. https://doi.org/10.1590/S0004-282X2011000500003

5. Palm A, Midgren B, Theorell-Haglöw J, Ekström M, Ljunggren M, Janson C, Lindberg E (2018) Factors influencing adherence to continuous positive airway pressure treatment in obstructive sleep apnea and mortality associated with treatment failure - a national registry-based cohort study. Sleep Med 51:85-91. https://doi.org/ 10.1016/j.sleep.2018.07.007

6. Catcheside PG (2010) Predictors of continuous positive airway pressure adherence. F1000 Med Rep. https://doi.org/10.3410/M270

7. Fox N, Hirsch-Allen A, Goodfellow E, Wenner J, Fleetham J, Ryan CF, Kwiatkowska M, Ayas NT (2012) The impact of a telemedicine monitoring system on positive airway pressure adherence in patients with obstructive sleep apnea: a randomized controlled trial. Sleep. 35:477-481. https://doi.org/10.5665/sleep.1728

8. Rotenberg BW, Murariu D, Pang KP (2016) Trends in CPAP adherence over twenty years of data collection: a flattened curve. $J$ Otolaryngol Head Neck Surg 45(1). https://doi.org/10.1186/ s40463-016-0156-0

9. Woehrle H, Graml A, Weinreich G (2010) Adherence to CPAP therapy. Somnologie. 14(2):135-139. https://doi.org/10.1007/ s11818-010-0470-0

10. Phillips BA (2008) Early CPAP use identifies subsequent adherence to CPAP therapy. Yearb Pulm Dis 2008:278-279. https://doi. org/10.1016/S8756-3452(08)70706-0

11. Sawyer AM, Gooneratne NS, Marcus CL, Ofer D, Richards KC, Weaver TE (2011) A systematic review of CPAP adherence across age groups: clinical and empiric insights for developing CPAP adherence interventions. Sleep Med Rev 15(6):343-356. https://doi. org/10.1016/j.smrv.2011.01.003

12. Shapiro GK, Shapiro CM (2010) Factors that influence CPAP adherence: an overview. Sleep Breath 14(4):323-335. https://doi.org/ 10.1007/s11325-010-0391-y
13. Sawhney P, Modi AC, Jenkins TM, Zeller MH, Kollar LM, Inge TH, Xanthakos SA (2013) Predictors and outcomes of adolescent bariatric support group attendance. Surg Obes Relat Dis 9(5):773779. https://doi.org/10.1016/j.soard.2013.03.016

14. Clancy DE, Huang P, Okonofua E, Yeager D, Magruder KM (2007) Group visits: promoting adherence to diabetes guidelines. J Gen Intern Med 22(5):620-624. https://doi.org/10.1007/s11606007-0150-3

15. Hollander SA, McDonald N, Lee D, May LJ, Doan LN, Kaufman BD, Rosenthal DN (2015) Group visits in the pediatric heart transplant outpatient clinic. Pediatr Transplant 19(7):730-736. https:// doi.org/10.1111/petr.12574

16. Rathbun RC, Farmer KC, Stephens JR, Lockhart SM (2005) Impact of an adherence clinic on behavioral outcomes and virologic response in treatment of HIV infection: a prospective, randomized, controlled pilot study. Clin Ther 27(2):199-209. https://doi.org/10. 1016/j.clinthera.2005.02.010

17. Manni R, Politin L, Ratti MT, Tartara A (1999) Sleepiness in obstructive sleep apnea syndrome and simple snoring evaluated by the Epworth Sleepiness Scale. J Sleep Res 8:319-320. https://doi.org/ 10.1046/j.1365-2869.1999.00166.x

18. Brown L, Tang X, Hollman R (2014) The structure of social exchange in self-help support groups: development of a measure. $\mathrm{Am}$ $J$ Community Psychol 53(1-2):83-95. https://doi.org/10.1007/ s10464-013-9621-3

19. Lettieri CJ, Walter RJ (2013) Impact of group education on continuous positive airway pressure adherence. J Clin Sleep Med 9(6): 537-541. https://doi.org/10.5664/jcsm.2742

20. Likar LL, Panciera TM, Erickson AD, Rounds S (1997) Group education sessions and compliance with nasal CPAP therapy. Chest. 111(5):1273-1277. https://doi.org/10.1378/chest.111.5. 1273

Publisher's note Springer Nature remains neutral with regard to jurisdictional claims in published maps and institutional affiliations. 